\title{
Histopathology, parasite density and cell phenotypes of the popliteal lymph node in canine visceral leishmaniasis
}

\author{
Rodolfo Cordeiro Giunchetti ${ }^{\mathrm{a}, \mathrm{b}, *}$, Olindo Assis Martins-Filho ${ }^{\mathrm{c}}$, \\ Cláudia Martins Carneiro ${ }^{\mathrm{a}, \mathrm{d}}$, Wilson Mayrink ${ }^{\mathrm{e}}$, Marcos José Marques ${ }^{\mathrm{f}}$, \\ Washington Luiz Tafuri ${ }^{\mathrm{a}}$, Rodrigo Corrêa-Oliveira ${ }^{\mathrm{b}}$, Alexandre Barbosa Reis ${ }^{\mathrm{a}, \mathrm{b}, \mathrm{d}, *}$ \\ a Laboratório Imunopatologia, Núcleo de Pesquisas em Ciências Biológicas/NUPEB, Instituto de Ciências Exatas e Biológicas, \\ Universidade Federal de Ouro Preto, Ouro Preto, Minas Gerais, Brazil \\ ${ }^{\mathrm{b}}$ Laboratório de Imunologia Celular e Molecular, Instituto de Pesquisas René Rachou, Fundação Oswaldo Cruz, Belo Horizonte, \\ Minas Gerais, Brazil \\ ${ }^{\mathrm{c}}$ Laboratório de Doença de Chagas, Instituto de Pesquisas René Rachou, Fundação Oswaldo Cruz, Belo Horizonte, \\ Minas Gerais, Brazil \\ ${ }^{\mathrm{d}}$ Departamento de Análises Clínicas, Escola de Farmácia, Universidade Federal de Ouro Preto, Ouro Preto, Minas Gerais, Brazil \\ ${ }^{\mathrm{e}}$ Laboratório de Leishmanioses, Departamento de Parasitologia, Instituto de Ciências Biológicas, \\ Universidade Federal de Minas Gerais, Belo Horizonte, Minas Gerais, Brazil \\ ${ }^{\mathrm{f}}$ Laboratório de Biologia Molecular e Biotecnologia, Universidade Federal de Alfenas, Alfenas, Minas Gerais, Brazil
}

Received 6 May 2007; received in revised form 16 July 2007; accepted 18 July 2007

\begin{abstract}
While enlargement of popliteal lymph nodes (LN) is frequently described in canine visceral leishmaniasis (CVL), there are few histopathologic studies of lymph nodes during this chronic immunopathological condition. Besides a detailed histopathologic analysis, we have characterized the parasite load and major immunophenotypic features of the LN in Leishmania (Leishmania) chagasi-infected dogs. Our major histopathological findings highlight that hypertrophy/hyperplasia of LN cortical and medullary zones was the principal characteristic observed in asymptomatic dogs (AD), whereas atrophy of LN cortical zone was predominant in symptomatic animals (SD). The LN parasite density detected by anti-Leishmania immunohistochemical assay or expressed as Leishman Donovan Units was also highly correlated with the skin parasitism, the most reliable parameter to decode the clinical status of CVL. The major LN immunophenotypic changes during ongoing CVL were an increased frequency of T-lymphocytes, particularly CD8 ${ }^{+} \mathrm{T}$-cells, upregulation of MHC-II expression by lymphocytes and decreased levels of $\mathrm{CD} 21^{+} \mathrm{B}$-cells. Our findings further demonstrated that changes in the LN B-lymphocyte compartment exhibited a negative correlation with the skin parasite load. Conversely, we also showed evidence for a positive association between skin parasitism and LN T-cell-mediated immunity, suggesting that T-cells, especially CD8 ${ }^{+}$ lymphocytes, may have a Type-2 immunological profile in this lymphoid tissue in response to CVL.
\end{abstract}

(C) 2007 Elsevier B.V. All rights reserved.

Keywords: Canine visceral leishmaniasis; Leishmania chagasi; Lymph node; Histopathology; Parasitism; Lymphocyte subsets; Flow cytometry

\footnotetext{
* Corresponding authors at: Laboratório de Imunopatologia, Núcleo de Pesquisas em Ciências Biológicas, Universidade Federal de Ouro Preto, Campus Universitário, Morro do Cruzeiro, Ouro Preto CEP 35400-000, MG, Brazil. Tel.: +55 313559 1694;

fax: +553135591680.

E-mail addresses: giunchetti@nupeb.ufop.br (R.C. Giunchetti), alexreis@nupeb.ufop.br (A.B. Reis).
}

\section{Introduction}

Leishmania (Leishmania) chagasi [syn Leishmania (Leishmania) infantum] infection is one of the most relevant zoonotic protozoa infection that affects humans and dogs in Europe and Latin America (Desjeux, 2004). 
Canine visceral leishmaniasis (CVL) is highly relevant from the epidemiological stand point, with an increasing incidence in recent decades. Indeed, in endemic areas of Brazil, elimination of infected dogs was associated with decreased prevalence of human disease (Alencar, 1961; Palatnik-de-Sousa et al., 2001). It has been postulated that the intense cutaneous parasitism reported in infected dogs, including asymptomatic carriers (Abranches et al., 1991; Giunchetti et al., 2006), represents the major feature that assign the dogs as the principal domestic reservoir for human infection (Deane and Deane, 1962; Abranches et al., 1991).

Although CVL is known to be a severe systemic disease, there are few studies describing detailed histopatological features of distinct host compartments affected by the parasite. We have previously described that symptomatic dogs present an intense diffuse dermal inflammatory infiltrate and high parasitic burden when compared to the asymptomatic dogs (Giunchetti et al., 2006). Moreover, we have also reported that symptomatic dogs display high frequency of hypertrophy/ hyperplasia of Küpffer cells and enhanced hepatic parasitism when compared to asymptomatic carriers (Giunchetti et al., 2007). Despite the fact that generalized lymphadenomegaly is a classical clinical feature frequently described in CVL, the histological aspects underlying the lymph node (LN) pathological condition is still poorly investigated (Keenan et al., 1984; Tafuri et al., 2001; Lima et al., 2004). It has been demonstrated that hypertrophy/hyperplasia of cortical and medullary zones are the most relevant findings, with no specific lymph node lesions in asymptomatic (AD), oligosymptomatic (OD) or symptomatic animals (SD), suggesting that LN immunopathological condition is a common characteristic of diffuse chronic inflammation despite the clinical form of the disease (Lima et al., 2004).

Although LN are one of the most relevant lymphoid tissues involved in the parasite-host interface during the stages of $L$. chagasi infection, the cellular and molecular immune response in the $\mathrm{LN}$ is still poorly elucidated. We hypothesize that the major histopathological changes in LN during CVL may reflect not only the profile of the host's immune response but also the parasite burden intensity throughout $L$. chagasi infection. Aiming to further characterize the immunological events that take place during CVL, we have performed a detailed investigation focusing on major LN histopathological, parasitological and immunological aspects. Our findings highlight that LN histopathological features previously described may reflect the overall tissue parasitism and have close association with the T-cell subset distribution in the popliteal lymph node. Together, our findings revealed a positive association between skin parasitism and the LN T-cell mediated immunity, suggesting that T-cells, especially $\mathrm{CD}^{+}$lymphocytes, may play a distinct role in this lymphoid tissue in addition to the previously described immunoprotective function of $\mathrm{CD} 8^{+} \mathrm{T}$-cells in response to CVL.

\section{Materials and methods}

\subsection{Animals}

Thirty-four mixed-breed adult dogs of both genders, 2-6 years old, were selected from the Control Zoonosis Center in Belo Horizonte City Council, Minas Gerais State, Brazil, in an endemic area for CVL. They were maintained in the kennels of the Institute of Biological Sciences of Federal University of Minas Gerais. Clinical pre-selection was carried out in the latter location. The dogs used in this study were stray or domiciled mongrel dogs, selected based on their serological results on indirect immunofluorescence assay test (IFAT), used as a 'gold standard' immunological test for diagnosis of CVL. Animals presenting indirect immunofluorescence assay test (IFAT) titer $\geq 1: 40$ and positive parasitological diagnosis to Leishmania in at least one tissue smear (bone marrow, ear, skin, spleen, liver or popliteous lymph node) were enrolled into the group of infected dogs (ID). Animals with negative IFAT results at 1:40 and negative parasitological tests to Leishmania were included as uninfected control (UD).

This study was approved by the Ethical Committee for the use of Experimental Animals of the Federal University of Minas Gerais, Brazil (CETEA).

\subsection{Clinical evaluation}

Leishmania (Leishmania) chagasi naturally infected dogs (ID = 26) were clinically sub-divided according to Mancianti et al. (1988) based on the presence/absence of infection signs: asymptomatic ( $\mathrm{AD}, n=8)$, with no suggestive signs of disease; oligosymptomatic (OD, $n=8$ ), with a maximum of three clinical signs including opaque bristles and/or localized alopecia and/or moderate loss of weight; symptomatic (SD, $n=10)$, with characteristic clinical signs of visceral leishmaniasis, such as cutaneous lesions, onychogriphosis, opaque bristles, severe loss of weight, apathy and keratoconjunctivitis; and uninfected dogs (UD, $n=8$ ), animals who were negative on serological and parasitological examination for Leishmania. 


\subsection{Lymph node and ear skin specimen collection}

Lymph node and ear skin specimen collection were carried out after euthanasia of the dogs with a barbiturate anesthesia (Thiopental ${ }^{\mathbb{R}}$ at $30 \mathrm{mg} / \mathrm{kg}$ of body weight). LN fragments were stored at room temperature, in $10 \%$ neutral buffered formalin for routine histological procedures by hematoxylin-eosin staining (lymph node) and anti-Leishmania immunohistochemical (lymph node and ear skin) examinations. Lymph node and ear skin imprints were performed on two microscopic slides and were air-dried. Samples were fixed in methanol, stained with Giemsa, and examined under optical microscopy for the identification of Leishmania amastigote forms.

Lymph node sections $(5 \mu \mathrm{m})$ were stored on ice, up to $12 \mathrm{~h}$, in sterile RPMI-1640 for immunophenotyping by flow cytometry.

\subsection{Histological evaluation}

The hypertrophy/hyperplasia of cortical and medullary zones patterns were evaluated through routine histological HE-staining of $5 \mu \mathrm{m}$ sections under optical microscopy. The hypertrophy/hyperplasia of cortical zone was evaluated by semi-quantitative analysis of major morphological aspects, including number of cells and germinal centers morphology, specifically the presence of large nuclei, branched chromatin and prominent nucleoli. The hypertrophy/hyperplasia of medullary zone was also evaluated by a semiquantitative analysis of the number of plasma cells, macrophages and lymphocytes. Histological aspects were further graded as atrophy or absence of hypertrophy/hyperplasia $(-)$; light $(+)$; moderate $(++)$ and intense hypertrophy/hyperplasia (+++).

\subsection{Parasite load assessment}

Giemsa-stained LN impression smears were examined under optical microscopy to determine the parasite density expressed as "Leishman Donovan Units" (LDU) as described by Stauber (1956) with some modifications. Briefly, Leishmania amastigote stages were counted and the results expressed as LDU index, equivalent to the number of amastigotes per 1000 nucleated cells. Parasite density assessed by LDU was categorized into tertiles, according Reis et al. (2006a) modified, as absent, $\mathrm{LDU}=0$; low, $\mathrm{LDU}=1-2$ and 1-9; medium, LDU $=3-24$ and 10-130 and high parasitism, LDU $=25-616$ and 131-7246 for lymph node and ear skin, respectively.
Additionally, parasite density was also evaluated by anti-Leishmania immunohistochemistry (IHC), as described by Tafuri et al. (2004). Briefly, heterologous hyperimmune serum from a $L$. chagasi naturally infected dog with IFAT titers $\geq 1: 1280$, diluted $1: 100$ in $0.01 \mathrm{M}$ PBS, was used as primary antibody. Following incubation and wash procedures, slides were treated with biotinylated goat anti-mouse and anti-rabbit antibody (Link-DAKO, LSAB2 Kit, Catalog \# KO675-1; Carpinteria, CA, USA), that display cross-reactivity with dog serum immunoglobulins, as previously reported by Tafuri et al. (2004) and then incubated with the streptavidin-peroxidase complex (DAKO, LSAB2 Kit, Catalog \# K0675-1; Carpinteria, CA, USA). The reaction was developed with a $0.024 \%$ diaminobenzidine (DAB; Sigma, St. Louis, USA) solution and $0.16 \%$ hydrogen peroxide $40 \%$ (v/v). Slides were then dehydrated, cleared, counter-stained with Harris' hematoxylin, and mounted with coverslips. Semi-quantitative analysis was performed under optical microscopy, and parasitism assessed as the number of immunolabeled amastigotes parasites and expressed as the mean number of amastigotes observed in five $400 \times$ fields. Parasite density detected by IHC was graded as absent $(-)$, low $(+)$, medium (++) and high (+++) parasitism corresponding to $0,1-2,3-24$ and $>24$ amastigotes, respectively.

\subsection{Flow cytometry analysis}

\subsubsection{Isolation of LN mononuclear cells}

Lymph node fragments $(5 \mathrm{~mm})$ were immersed in cold RPMI-1640 in a Petri dish and placed on ice. The tissue was minced in tissue grinder and transferred to $2 \mathrm{ml}$ of RPMI-1640 (GIBCO, Grand Island, NY, USA). The cell suspension was then filtered using stainless steel gauze to obtain a single cell suspension. The mononuclear LN cells were isolated by differential centrifugation $(800 \times g$ for $40 \mathrm{~min}$ at room temperature(RT)) on Ficoll-Hypaque gradient (Histopaque ${ }^{\mathbb{R}}$ 1.077-Sigma Chemical Co.). The cell suspension was washed twice in RPMI-1640 and resuspended to the concentration of $10^{7}$ cells $/ \mathrm{ml}$.

\subsubsection{Immunophenotyping procedures}

Analysis of LN mononuclear cell phenotypes was performed by flow cytometry, as previously described by Reis et al. (2005). Briefly, $1 \mathrm{ml}$ of cell suspension was submitted to pre-fixation by slow addition of $5 \mathrm{ml}$ of lysing pre-fix solution (FACS lysing solution (FLS) Becton Dickinson, Moutain View, CA) followed by incubation for $10 \mathrm{~min}$ at room temperature (RT). After centrifugation $(450 \times g$, for $10 \mathrm{~min}$, at $\mathrm{RT})$, the pellet 
was resuspended in $500 \mu l$ phosphate buffered saline supplemented with $10 \%$ of fetal bovine serum. In 96 wells "U" bottom plate (LIMBRO Biomedicals Inc., Aurora, Ohio), $30 \mu \mathrm{l}$ of pre-fixed leukocyte suspension (approximately $6 \times 10^{5}$ cells) were incubated at RT for $30 \mathrm{~min}$ in the dark, with $30 \mu \mathrm{l}$ anti-canine cell surface markers antibodies. Monoclonal antibodies (MAbs) that define canine cell phenotypes included purified rat antiThy-1 (Rat-IgG2b: Clone YKIX337.217), anti-CD5
(Rat-IgG2a: Clone YKIX322.3), anti-CD4 (Rat-IgG2a: Clone YKIX302.9), anti-CD8 (Rat-IgG1: Clone YCATE55.9), anti-MHC-II (Rat-IgG2b: Clone YKIX334.2), and FITC-labeled mouse anti-CD21 (Mouse-IgG1: Clone IOBla). These antibodies were used on indirect and direct immunofluorescence procedures. Unlabeled MAbs used in this study were purchased from SEROTEC (Oxford, UK) and antiCD21 from Immunotech Co. (Marseilles, France).
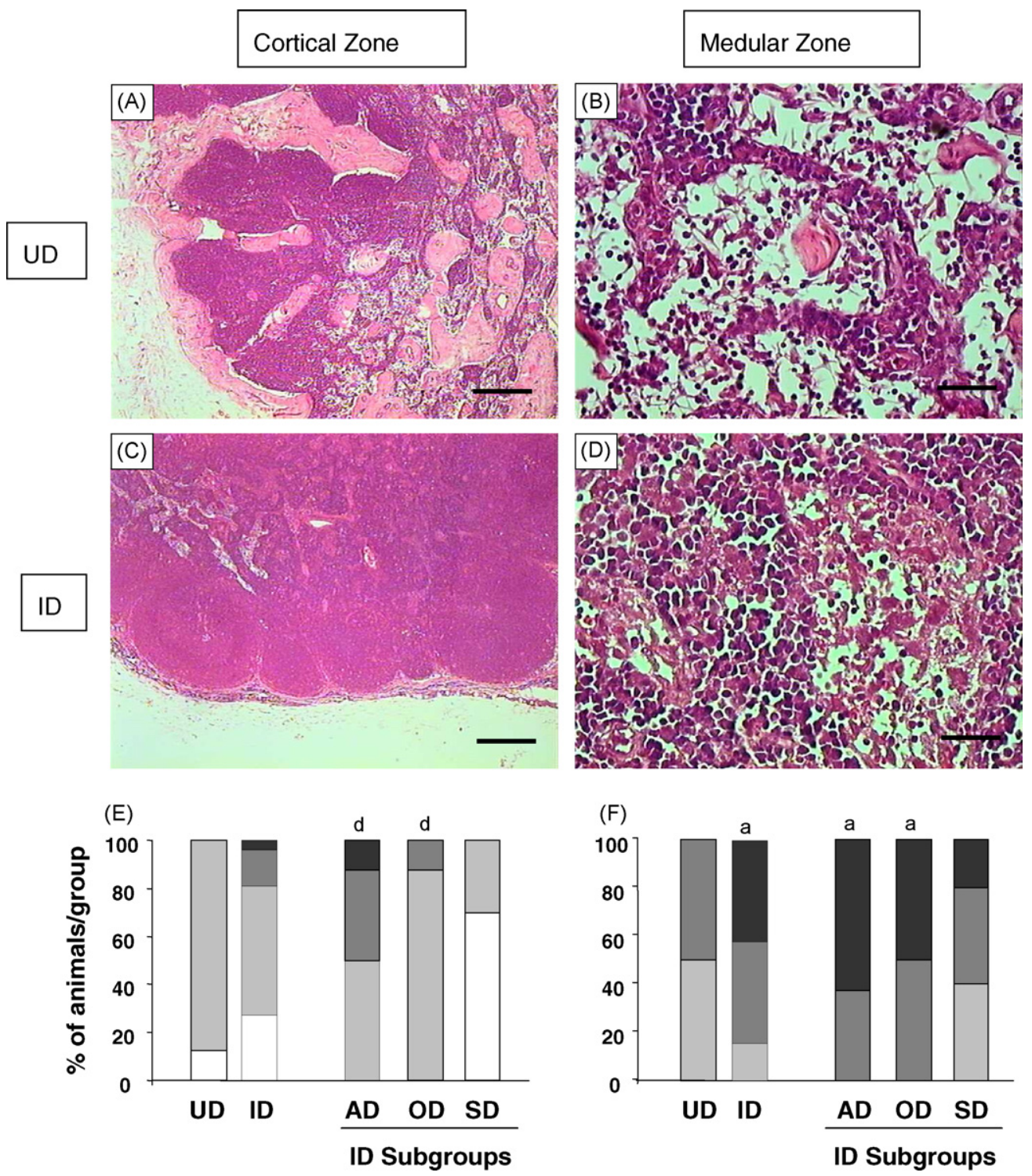

Fig. 1. Major histopathological features of popliteal lymph node from uninfected dogs (UD) and Leishmania chagasi-infected dogs (ID). Photomicroscopy of haematoxylin-eosin stained specimens highlighting the histological aspect of cortical $(\mathrm{A}$; bar $=250 \mu \mathrm{m})$ and medullary regions $(B ;$ bar $=25 \mu \mathrm{m})$ from UD in contrast to hypertrophy/hyperplasia of cortical $(C$; bar $=250 \mu \mathrm{m})$ and medullary $(\mathrm{D} ;$ bar $=25 \mu \mathrm{m})$ regions observed in ID $($ bar $=25 \mu \mathrm{m})$. The graphs illustrate the intensity of cortical $(\mathrm{E})$ and medullary hypertrophy/hyperplasia $(\mathrm{F})$ in popliteous lymph nodes from UD and ID sub-groups (AD, asymptomatic dogs; OD, oligosymptomatic dogs; SD, symptomatic dogs). $(\square)=$ atrophy or absence of hypertrophy/ hyperplasia; $(\square)$ light; $(\square)=$ moderate and $(\square)=$ intense hypertrophy/hyperplasia. Significant results $(P<0.05)$ are represented by letters "a" and "d" as compared to UD and SD, respectively. 
When purified MAbs were used, the cells were incubated at the same conditions in the presence of $60 \mu \mathrm{l}$ of previous diluted FITC-conjugated sheep antirat IgG antibody. Before flow cytometric data collection and analysis, labeled cells were fixed for $30 \mathrm{~min}$ with $200 \mu \mathrm{l}$ of FACS FIX solution (10.0 g/l paraformalde- hyde; $10.2 \mathrm{~g} / \mathrm{l}$ sodium cacodylate and $6.65 \mathrm{~g} / \mathrm{l}$ sodium chloride, $\mathrm{pH}$ 7.2).

Results were expressed as percentage of gated lymphocytes for Thy- $1^{+}$and $\mathrm{CD} 5^{+}$T-cells; CD $21^{+} \mathrm{B}-$ cells; $\mathrm{CD}^{+}$and $\mathrm{CD}^{+}$T-cell subsets. Data regarding lymphocyte activation status were presented as the

\section{Parasite Load}

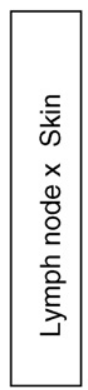

(A)
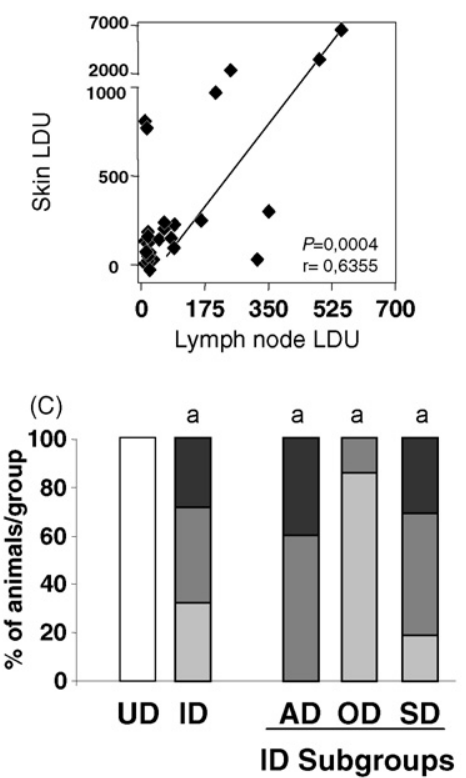

$\square=0 \square=01-02 \square=03-24 \square=25-616$

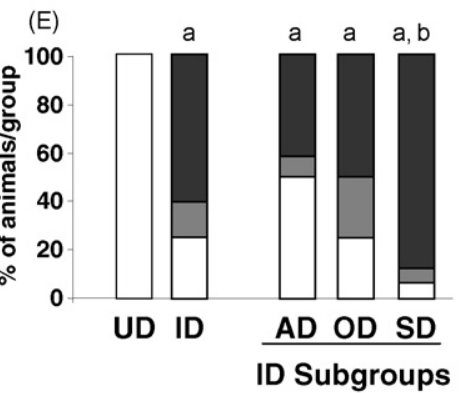

(B)
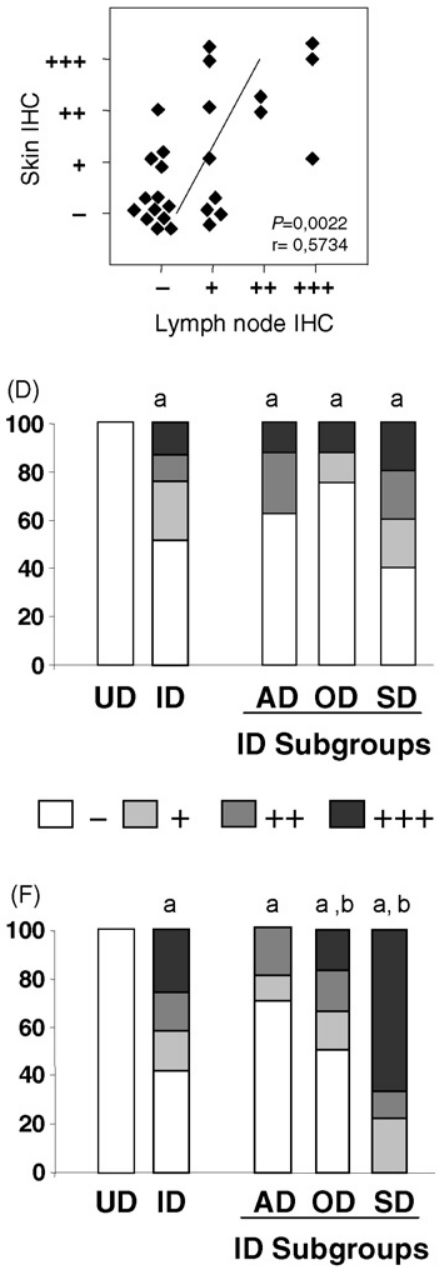

$L D U \times I H C$
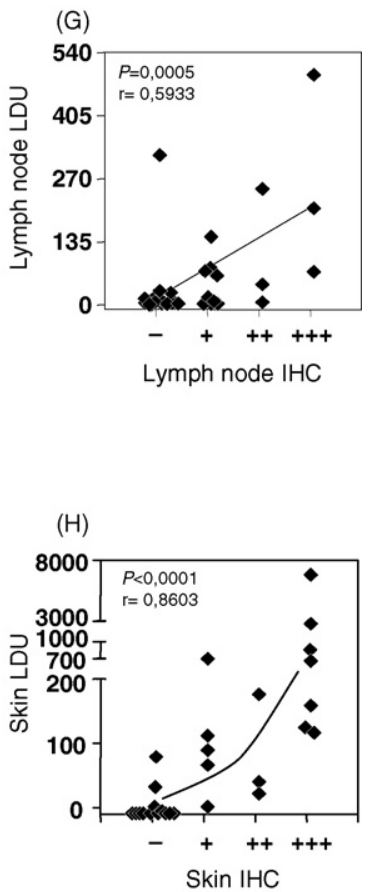

$\square=0 \square=01-09 \square=10-130 \square=131-7246$

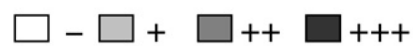

Fig. 2. Parasite density features in popliteal lymph node and ear skin expressed as Leishman Donovan Units (LDU) or anti-Leishmania immunohistochemical assay (IHC). Correlation analysis demonstrated direct association between parasite load in popliteal lymph node and ear skin, despite the methodology, LDU (A) and IHC (B). Popliteal lymph node and skin parasite load was further classified as absent ( $\square$ ); low ( $\square$ ); medium ( $\square$ ) and high parasitism ( $\square$ ) and report for all clinical groups evaluated. L. chagasi-infected dogs (ID) were additionally sub-grouped as asymptomatic (AD), oligosymptomatic (OD) and symptomatic dogs (SD). Statistically significant differences are highlighted by the letters "a" and "b" as compared to UD and AD, at $P<0.05$. Correlation analysis further confirmed the degree of association between LDU and IHC. Data are expressed as scattering of individual values and frequency columns graphs for popliteal lymph node and ear skin parasite load. Spearman correlation indexes ( $r$ and $P$ values) are shown on graphs. Connecting lines illustrates the correlation indexes. 
mean fluorescence intensity for MHC-II expression by gated cells.

\subsection{Statistical analysis}

Statistical analysis was performed using the Prism 4.1 software package (Prism Software, Irvine, CA, USA). Mann-Whitney test was used for comparative analysis of major histopathological and parasitological findings between UD and ID. Kruskal-Wallis followed by Dunns' tests were used for multiple comparisons of major histopathological and parasitological features $(\mathrm{UD} \times \mathrm{AD} \times \mathrm{OD} \times \mathrm{SD})$. Analysis of $\mathrm{LN}$ immunophenotypes was performed by the Mann-Whitney test to compare UD and ID groups. Spearman test was performed for different correlations strategies. Differences were considered significant when the probabilities of equality, $P$ values were $\leq 0.05$.

\section{Results}

\subsection{Hypertrophy/hyperplasia of LN cortical zone is the major characteristic of asymptomatic dogs whereas atrophy of LN cortical zone is the predominant histopathological finding in symptomatic animals}

Histological analysis was performed by the routine hematoxylin-eosin (HE) procedure. Our findings revealed that depletion of follicular structures and lymphocytes which have been replaced mainly by macrophages are the major LN histological features at the cortical zone. The main findings observed in the LN cortical zone in uninfected and naturally $L$. chagasiinfected dogs are shown in Fig. 1 (left panels). Distinct from the histological aspect observed for UD (Fig. 1A) hypertrophy/hyperplasia of cortical region was commonly observed in infected dogs (Fig. 1C). Our findings demonstrated intense hypertrophy/hyperplasia of cortical region in $\mathrm{AD}$ and $\mathrm{OD}$ group as compared to $\mathrm{SD}$. On the other hand, atrophy of LN cortical zone was predominantly observed in SD (Fig. 1E).

\subsection{Hypertrophy/hyperplasia of LN medullary zone was also the hallmark of asymptomatic disease}

Histological aspects of cords and sinuses of LN medullary regions from uninfected and $L$. chagasiinfected dogs were evaluated by routine hematoxylineosin (HE) procedure and the major findings are presented in Fig. 1 (right panels). In contrast to the histology seen in UD (Fig. 1B), cellular infiltrates in the medullary region of LN from L. chagasi-infected dogs were mainly characterized by the hypertrophy/hyperplasia of plasmacytes when compared to the frequency of macrophages and lymphocytes (Fig. 1D). Data analysis demonstrated that ID presented higher LN medullary reactivity as compared to UD (Fig. 1F). Furthermore, only AD and OD showed marked LN medullary reactivity as compared to UD, characterized by prominent hypertrophy/hyperplasia of cords and sinus (Fig. 1F).

\subsection{Despite the high correlation between skin and $L N$ parasite burden observed in L. chagasi-infected dogs, the skin parasitism was the most reliable indicator of the clinical status of CVL}

Lymph node and ear skin parasite load were assessed using two distinct approaches including Giemsa-stained microscopy to determine the Leishman Donovan Units (LDU) and anti-Leishmania immunohistochemical analysis (IHC; Fig. 2). Data analysis demonstrated positive correlation between the parasitism assessed by these different approaches (IHC and LDU; Fig. 2A and B) in both compartments (Fig. 2G and H). Additional analysis was performed by scoring popliteal lymph node and skin parasite load as absent, low, medium or high and reported for all clinical groups evaluated (Fig. 2C-F). L. chagasi naturally infected dogs (ID) were additionally sub-grouped as asymptomatic (AD), oligosymptomatic (OD) and symptomatic dogs (SD). Despite the fact that no difference was observed in LN parasite burden evaluation of different clinical groups (Fig. 2C and D), the ear skin parasite load revealed higher parasitism, expressed as LDU, in SD group when compared to both UD and AD groups (Fig. 2E). Analysis of parasite load by IHC showed better correspondence between parasite load and clinical status of CVL, highlighting the presence of higher ear skin parasite density in SD and OD when compared to UD and AD (Fig. 2F).

\subsection{Increased frequency of T-lymphocytes,} particularly $C D 8^{+}$cells, in addition to decreased levels of $\mathrm{CD} 2 \mathrm{I}^{+} \mathrm{B}$-cells and up-regulation of $\mathrm{MHC}$ II expression by lymphocytes represented the major $L N$ immunophenotypic changes during ongoing $C V L$

Immunophenotypic analysis of $\mathrm{LN}$ cells was performed by direct and indirect flow cytometry immunofluorescence. The major LN immunophenotypic features are presented in Fig. 3. Data analysis 
demonstrated higher frequency of T-lymphocytes (both Thy $-1^{+}$and $\mathrm{CD}^{+}$), mainly due the enhanced levels of $\mathrm{CD}^{+}$T-cells in addition to a lower percentage of $\mathrm{CD} 21^{+}$B-cells in naturally infected $\operatorname{dogs}$ (ID) in comparison to uninfected controls (UD) (Fig. 3A, C and $\mathrm{B}$, respectively). No changes in the percentage of $\mathrm{LN}$ $\mathrm{CD}^{+}{ }^{+}$T-cells were observed between ID and UD (Fig. 3C).
Analysis of the activation status of $\mathrm{LN}$ cells was performed and the relative mean fluorescence intensity of the cell surface activation marker (MHC-II) is presented in Fig. 3D. Data analysis demonstrated differential expression of MHC-II in ID, leading to significantly increased fluorescence intensity on gated lymphocytes when compared to UD (Fig. 3D).
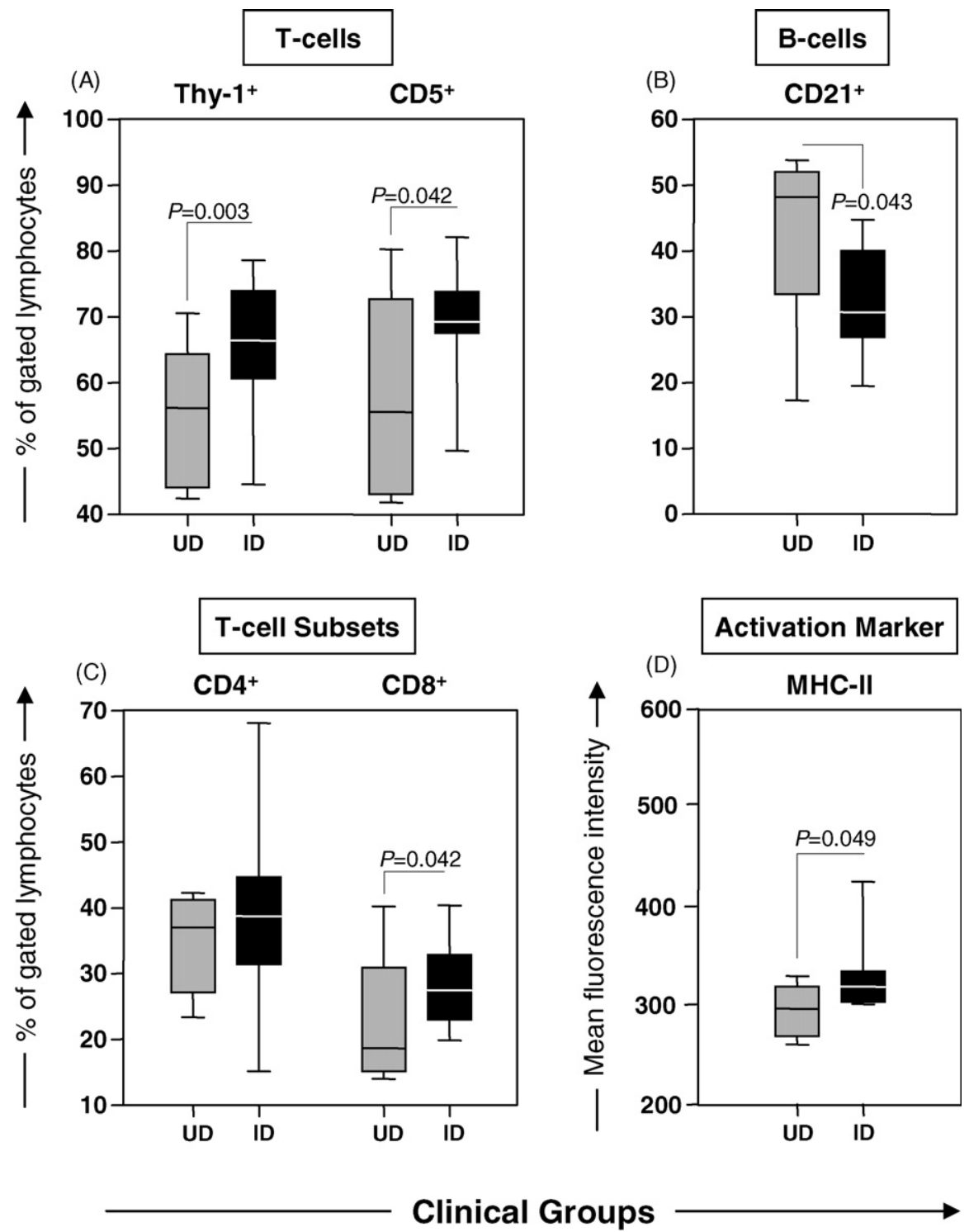

Fig. 3. Immunophenotypic profile of popliteal lymph node from uninfected dogs $(\square)$ and L. chagasi-infected dogs

The results are expressed in box-plot format showing the percentage of gated lymphocytes for Thy- $1^{+}$and $\mathrm{CD} 5^{+} \mathrm{T}$-cells $(\mathrm{A})$; CD2 $1^{+} \mathrm{B}-\mathrm{cells}(\mathrm{B})$; CD $4^{+}$and $\mathrm{CD} 8^{+} \mathrm{T}$-cell subsets (C). Activation status was presented as the mean fluorescence intensity for MHC-II by gated lymphocytes (D). The box stretches from the lower hinge (defined as the 25 th percentile) to the upper hinge (the 75 th percentile) and therefore contains the middle half of the score in the distribution. The median is shown as a line across the box. Therefore 1/4 of the distribution is between this line and the bottom or the top of the box. Significant differences at $P<0.05$ are highlighted by connecting lines. 


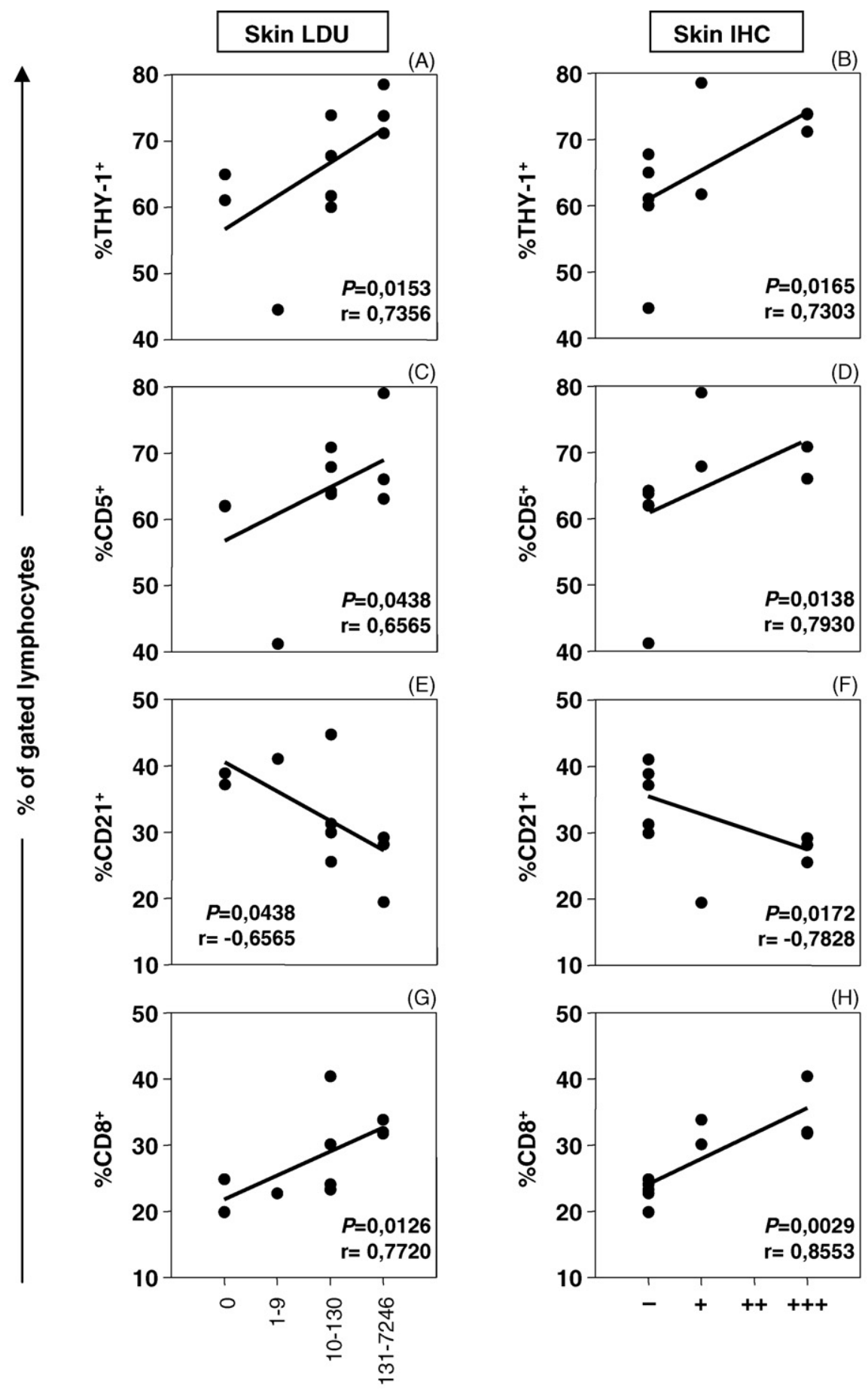

\section{Skin Parasite Load}

Fig. 4. Correlation between popliteal lymph nodes immunophenotypes and ear skin parasite load from L. chagasi-infected dogs. Parasite load are expressed as Leishman Donovan Units (LDU) (left panels) and anti-Leishmania immunohistochemical assay (IHC) (right panels), categorized as absent, LDU $=0$ or IHC $(-=0)$; low, LDU (1-9) or IHC (+ = 1-2); medium, LDU (10-130) or IHC (++ = 3-24) and high parasitism, LDU (131$7246)$ or IHC $(+++=>24)$. Cell phenotypes are expressed as percentage of Thy- $1^{+}(\mathrm{A}, \mathrm{B}), \mathrm{CD}^{+}(\mathrm{C}, \mathrm{D}), \mathrm{CD} 21^{+}(\mathrm{E}, \mathrm{F})$ and CD8 ${ }^{+}(\mathrm{G}, \mathrm{H})$ cells within gated lymphocytes. Data are shown as scattering of individual values. Spearman correlation indexes $(r)$ at $P<0.05$ are shown on graphs. Connecting lines illustrate the correlation indexes. 
3.5. Positive association between the ear skin parasitism and LN T-cell mediated immunity suggests that $L N C D 8^{+}$lymphocytes may have a distinctive role in the immune response during $C V L$

Attempting to investigate whether LN immunophenotypic features may be a reliable indicator of the ear skin parasite load, we have further characterized the level of association between these variables, as shown in Fig. 4. Data analysis demonstrated that despite a negative association between LN B-cells and ear skin parasitism was observed, a strong positive correlation between LN T-lymphocytes (both Thy- $1^{+}$and $\mathrm{CD} 5^{+}$), particularly $\mathrm{CD}^{+}$cells, are the hallmark of $L$. chagasi infection. Comparable correlation indexes were found using both parasitological approaches to detect the parasite burden, including LDU (left panels) and IHC (right panels). No significant correlation indexes were observed when ear skin parasite load was paired at individual levels with $\mathrm{CD} 4^{+}$cell frequency (data not shown).

\section{Discussion}

The lesions observed in L. chagasi-infected dogs are similar in many respect to those described for human visceral leishmaniasis, with major gross lesions including hepatosplenomegaly and lymphadenopathy (Keenan et al., 1984). Despite the significant changes observed in the lymphoid tissues in response to $L$. chagasi infection, there are few studies focusing on the LN histopathology during CVL (Keenan et al., 1984; Martinez-Moreno et al., 1993; Tafuri et al., 2001; Lima et al., 2004). In CVL, lymphadenopathy is defined as an increase in LN size (enlargement of LN), usually described as a localized or generalized alteration (Rogers et al., 1993). It has been demonstrated that LN from L. chagasi-infected dogs display chronic lymphadenitis, independently of the anatomical region analyzed, with hypertrophy/hyperplasia of cortical and medullary zones (Lima et al., 2004).

In the present study, we confirmed most of these previous observations, reporting that depletion of follicular structures and lymphocytes, which are mainly replaced by macrophages, are the major LN histopathogical features in the cortical zone during CVL. On the other hand, the hypertrophy/hyperplasia of plasma cells, compared to the frequency of lymphocytes and macrophages, has been identified as the most relevant histological finding in the medullary region. These features have been also demonstrated in human visceral leishmaniasis (Veress et al., 1977), experimental infections in hamsters (Corbett et al., 1992) and confirm previous reports in experimental and natural CVL (Keenan et al., 1984; Martinez-Moreno et al., 1993).

It has been suggested that no significant differences of LN histopathological features are observed between asymptomatic and symptomatic dogs (Lima et al., 2004). However, we have found that hypertrophy/hyperplasia of cortical and medullary zones are distinctly observed in $\mathrm{AD}$ and SD. While intense hypertrophy/hyperplasia of cortical zone is observed in AD and OD groups, atrophy of cortical zone is more frequent in SD group. Moreover, only AD and OD groups exhibited a higher intensity of medullary reactivity as compared to UD group. Our data suggests that the decreased number of cells in germinal centers might be due to immunoregulatory mechanisms, such as apoptosis, would be one of the most relevant events underlying the cortical atrophy as observed in SD group. Further investigations are still required to test this hypothesis. Taken together, these findings supported the hypothesis that an intense $\mathrm{LN}$ immune response is important in controlling the clinical status during ongoing CVL.

Aiming to better understand the events underlying the compartmentalized immune response in the $\mathrm{LN}$, we have performed a detailed analysis of $\mathrm{LN}$ parasite load and characterization of the phenotypic profile of major LN lymphocyte populations. Our previous paper reported that the skin is the major site of high parasite density during ongoing canine visceral leishmaniasis (Reis et al., 2006a,c). To further investigate the relationship between LN and skin we have performed a parasite load association. Analysis of parasite load in CVL in different tissues is a recent approach using antiLeishmania immunohistochemical and/or LDU index (Giunchetti et al., 2006, 2007; Lima et al., 2004; Reis et al., 2006a,b,c). Our findings demonstrated that despite a positive correlation between $\mathrm{LN}$ and skin parasitism, only skin parasitism was associated with the clinical status of CVL. Therefore, skin parasitism should be considered the best site for parasitological analysis as a reliable indicator of the severity of clinical disease in CVL. Indeed, we have previously reported that symptomatic dogs showed a large number of cutaneous changes exemplified by an intense inflammatory infiltrate, extracellular matrix changes and extreme parasite load (Giunchetti et al., 2006). The lack of association between the LN parasite burden scoring status and the clinical manifestation of CVL are in agreement with previous reports from Lima et al. (2004). Importantly, the SD group displayed lower frequency of negative IHC results in comparison to $\mathrm{AD}$ and OD (data not shown). Indeed, previous reports from 
Reis et al. (2006a), taking into account the frequency of distinct degrees of LN parasite density using LDU index, have demonstrated that the SD group presented a lower percentage of animals displaying low LN parasitism.

No previous investigation has reported on the LN immunophenotypic profile during $L$. chagasi infection. Our findings demonstrated that the LN lymphocyte population of L. chagasi-infected dogs (ID) is characterized by a significant increase in Thy $-1^{+}$and $\mathrm{CD}^{+}$T-cells, particularly $\mathrm{CD}^{+} \mathrm{T}$-cells, when compared to uninfected $\operatorname{dogs}$ (UD). $\mathrm{CD}^{+}$T-cells are thought to play an important role in the development of an effective immune response to Leishmania sp., possibly through cytotoxic mechanisms which act as potent protective events during CVL (Pinelli, 1997). Indeed, several studies have correlated the level of $\mathrm{CD}^{+}$T-cells with protection during $L$. chagasi infection (Reis et al., 2006b; Pinelli, 1997). Notorious of importance was our finding that $\mathrm{LN} \mathrm{CD} 8^{+} \mathrm{T}$-cells displayed a positive correlation with skin parasite load, with the highest levels of $\mathrm{CD}^{+}$T-cell observed in animals bearing the highest skin parasitism. Typically, high $\mathrm{CD}^{+}$T-cells counts have been observed in the peripheral blood of asymptomatic dogs and are associated with low bone marrow parasitism (Reis et al., 2006b). Therefore, we postulate that $\mathrm{LN} \mathrm{CD} 8^{+} \mathrm{T}-$ cells may present a distinct activation status during CVL, possibly associated with immunomodulatory or suppressor cell activity. In support of this hypothesis, Peruhype-Magalhães et al. (2006) showed in human active VL that circulating $\mathrm{CD} 8^{+} \mathrm{T}$-cells showed a mixed cytokine pattern characterized by elevated levels of both intracellular IFN- $\gamma$ and IL-10. Similar results implying a mixed pattern of cytokine mRNA in canine VL has been published (Lage et al., 2007). It is possible that a selective migration of Type- $2 \mathrm{CD}^{+} \mathrm{T}$-cells to the $\mathrm{LN}$, committed with the synthesis of IL-10, represents a relevant immunological feature for suppressing the cell mediated immune response observed in dogs with high parasite load. We hypothesize that the elevated functional activation state towards a Type- 2 immunological profile may account for the role of $\mathrm{LNCD}^{+} \mathrm{T}$-cells in $L$. chagasi-infected dogs. In addition, $\mathrm{CD}^{+}$cells may mediate protection not only through a quantitative enhancement of cell numbers but also through a qualitative change in functional capacity towards a Type-1 immune response that increases circulating Tcells migration to distinct host tissues. Specific studies focusing this issue, through characterization of the cytokine pattern of $\mathrm{LN} \mathrm{CD}^{+}$T-cells during CVL are currently under investigations in our laboratory.
In the present study, we have observed a significant decrease in $\mathrm{LN} \mathrm{CD} 21^{+}$B lymphocytes in ID as compared to UD group. Lower levels of $\mathrm{CD} 21^{+} \mathrm{B}$ lymphocytes have been also reported in the peripheral blood during CVL, especially in symptomatic dogs (Bourdoiseau et al., 1997; Reis et al., 2006b). The intense LN plasmacyte infiltration observed in our histopatological analysis is consistent with the hypothesis that B-cell migrates from peripheral blood to $\mathrm{LN}$ during CVL. Indeed, CD $21^{+} \mathrm{B}$ cell differentiation toward plasmacytes may lead to the loss of the CD21 cell marker and therefore lower $\mathrm{CD} 21^{+}$B-cell frequency in LN. Consistent with this hypothesis, our data revealed a negative correlation between the $\mathrm{LN}$ $\mathrm{CD} 21^{+} \mathrm{B}$-cell frequency and skin parasite load, since lower B-cell counts and higher parasite load are usually observed in SD and/or animals displaying higher antiLeishmania antibody levels (Reis et al., 2006a,b,c).

It has been proposed that increased expression of MHC-II may reflect an antigenic-priming-related immunological event (Reis et al., 2006b). The evaluation in lymphocyte activation status showed significant increase in expression of MHC-II in ID compared to UD. Additional analysis showed that the level of MHCII expression by LN lymphocytes was negatively associated with the skin parasite density evaluated as LDU index $(P<0.0368 / r=-0.7619)$. Together, our findings support the hypothesis that lymphocyte activation in the lymph nodes may favor cell migration and control of the parasite burden in parasitized organs. Consistent with this hypothesis, Reis and colleagues have demonstrated that asymptomatic dogs display enhanced activation status (expression of MHC-II) of circulating lymphocytes (Reis et al., 2006b) besides lower overall tissue parasitism (Reis et al., 2006a).

In conclusion, the histopathological profile of the asymptomatic disease was characterized by hypertrophy/hyperplasia of LN cortical zone and intense cell reactivity in LN medullary zone, with hypertrophy/ hyperplasia of cords and sinus. Cell phenotype analysis revealed decreased levels of $\mathrm{LN} \mathrm{CD} 21^{+} \mathrm{B}$-cells and an increased frequency of T-cells, particularly $\mathrm{CD} 8^{+} \mathrm{T}$ cells, associated with higher ear skin parasitism, suggesting a Type-2 immunological profile for $\mathrm{LN}$ $\mathrm{CD}^{+}{ }^{+}$T-cells. Further investigations focusing on $\mathrm{LN}$ cytokine profile, especially of $\mathrm{CD}^{+}$T-cells, are necessary to validate this hypothesis.

\section{Acknowledgments}

This work was supported by the $\mathrm{CNPq} / \mathrm{BR} /$ grant: 521124/98-0; FAPEMIG/BR/grant: CBB 901/06 and 
FIOCRUZ/PAPES IVB/2006. We are thankful to the Center for Zoonosis Control-Regional District of Belo Horizonte, Minas Gerais for the special dedication to this work. We also thank Dr. Erika Lamb of Microbiology and Immunology Department, USUHS, Bethesda, MD, USA for the critical reading of the manuscript, editorial suggestions and changes.

\section{References}

Abranches, P., Silva-Pereira, M.C.D., Conceição-Silva, F., SantosGomes, G.M., Jans, J.G., 1991. Canine leishmaniasis: pathological and ecological factors influencing transmission of infection. J. Parasitol. 77, 561-577.

Alencar, J.E., 1961. Profilaxia do kala-azar no Ceara. Brazil. Rev. Inst. Med. Trop. São Paulo 3, 175-180.

Bourdoiseau, G., Bonnefont, C., Magnol, J.P., Saint-Anré, I., Chabanne, L., 1997. Lymphocyte subset abnormalities in canine leishmaniasis. Vet. Immunol. Immunopathol. 56, 345-351.

Corbett, C.E., Pinto-Paes, P., Laurenti, R.A., Andrade Jr., M.D., Duarte, M.I.S., 1992. Histopathology of lymphoid organs in experimental leishmaniasis. Inst. J. Exp. Pathol. 73, 417-433.

Deane, L.M., Deane, M.P., 1962. Visceral leishmaniasis in Brazil. Geographical distribution and transmission. Rev. Inst. Med. Trop. São Paulo 4, 149-212.

Desjeux, P., 2004. Leishmaniasis: current situation and new perspectives. Comp. Immunol. Microbiol. Infect. Dis. 27, 305-318.

Giunchetti, R.C., Mayrink, W., Genaro, O., Carneiro, C.M., CorrêaOliveira, R., Martins-Filho, O.A., Marques, M.J., Tafuri, W.L., Reis, A.B., 2006. Relationship between canine visceral leishmaniosis and the Leishmania (Leishmania) chagasi burden in dermal inflammatory foci. J. Comp. Pathol. 135, 100-107.

Giunchetti, R.C., Mayrink, W., Carneiro, C.M., Corrêa-Oliveira, R., Martins-Filho, O.A., Marques, M.J., Tafuri, W.L., Reis, A.B., 2007. Histopathological and immunohistochemical investigations of the hepatic compartment associated with parasitism and serum biochemical changes in canine visceral leishmaniasis. Res. Vet. Sci., in press, doi:10.1016/j.rvsc.2007.04.020.

Keenan, C.N., Hendricks, L.D., Lightner, L., Johnson, A.J., 1984. Visceral leishmaniasis in a German shepherd dog II. Pathol. Vet. Pathol. 21, 80-86.

Lage, R.S., Oliveira, G.C., Buzek, S.C.U., Guerra, L.L., Giunchetti, R.C., Corrêa-Oliveira, R., Reis, A.B., 2007. Analysis of the cytokine profile in spleen cells from dogs naturally infected by Leishmania chagasi. Vet. Immunol. Immunopathol. 115, 135-145.

Lima, W.G., Michalick, M.S.M., Melo, M.N., Tafuri, W.L., Tafuri, Wg.L., 2004. Canine visceral leishmaniasis: a histopathological study of lymph nodes. Acta Trop. 92, 43-53.

Mancianti, F., Gramiccia, M., Gradoni, L., Pieri, S., 1988. Studies on canine leishmaniasis control I. Evolution of infection of different clinical forms of canine leishmaniasis following antimonils treatment. Trans. R. Soc. Trop. Med. Hyg. 82, 566-567.

Martinez-Moreno, A., Martinez-Cruz, M.S., Hernandez-Rodriguez, S., 1993. Immunological and histological study of T- and Blymphocyte activity in canine visceral leishmaniosis. Vet. Parasitol. 51, 49-59.
Palatnik-de-Sousa, C.B., Santos, W.R., França-Silva, J.C., da Costa, R.T., Reis, A.B., Palatnik, M., Mayrink, W., Genaro, O., 2001. Impact of canine control on the epidemiology of canine and human visceral leishmaniasis in Brazil. Am. J. Trop. Med. Hyg. 65, 510-517.

Peruhype-Magalhães, V., Martins-Filho, O.A., Prata, A., Siva, L.A., Rabello, A., Teixeira-Carvalho, A., Figueiredo, R.M., Guimarães-Carvalho, S.F., Ferrari, T.C.A., Van Weyenbergh, J., CorreaOliveira, R., 2006. Mixed inflammatory/regulatory cytokine profile marked by simultaneous raise of interferon-gamma and interleukin-10 and low frequency of tumour necrosis factoralpha monocytes are hallmarks of active human visceral leishmaniasis due to Leishmania chagasi infection. Clin. Exp. Immunol. 146, 124-132.

Pinelli, E., 1997. Cytokines in Canine Visceral Leishmaniasis. In: Schijns, V.E.C.J., Horzinek, M.C. (Eds.), Cytokines in Veterinary Medicine. Utrecht University, Netherlands, pp. 217-247.

Reis, A.B., Carneiro, C.M., Carvalho, M.G., Teixeira-Carvalho, A., Giunchetti, R.C., Mayrink, W., Genaro, L.L, Corrêa-Oliveira, R., Martins-Filho, O.A., 2005. Establishment of a microplate assay for flow cytometric assessment and it is use for the evaluation of age-related phenotypic changes in canine whole blood leukocytes. Vet. Immunol. Immunopathol. 103, 173-185.

Reis, A.B., Teixeira-Carvalho, A., Vale, A.M., Marques, M.J., Giunchetti, R.C., Mayrink, W., Guerra, L.L., Andrade, R.A., Corrêa-Oliveira, R., Martins-Filho, O.A., 2006a. Isotype patterns of immunoglobulins: hallmarks for clinical status and tissue parasite density in Brazilian dogs naturally infected by Leishmania (Leishmania) chagasi. Vet. Immunol. Immunopathol. 112, 102-116.

Reis, A.B., Teixeira-Carvalho, A., Giunchetti, R.C., Guerra, L.L., Carvalho, M.G., Mayrink, W., Genaro, O., Corrêa-Oliveira, R., Martins-Filho, O.A., 2006b. Phenotypic features of circulating leucocytes as immunological markers for clinical status and bone marrow parasite density in dogs naturally infected by Leishmania chagasi. Clin. Exp. Immunol. 146, 303-311.

Reis, A.B., Martins-Filho, O.A., Teixeira-Carvalho, A., Carvalho, M.G., Mayrink, W., França-Silva, J.C., Giunchetti, R.C., Genaro, O., Corrêa-Oliveira, R., 2006c. Parasite density and impaired biochemical/hematological status are associated with severe clinical aspects of canine visceral leishmaniasis. Res. Vet. Sci. 81, 68-75.

Rogers, K.S., Barton, C.L., Landis, M., 1993. Canine and feline lymph nodes. Part II. Diagnosis, evaluation and lymphadenopathy. Compendium 15, 1493-1501.

Stauber, L.A., 1956. Resistance to the Khartoum strain of Leishmania donovani. Rice Inst. Pamphlet. 45, 80-96.

Tafuri, W.L., De Oliveira, M.R., Melo, M.N., Tafuri, W.L., 2001. Canine visceral leishmaniasis: a remarkable histopathological picture of one case report from Brazil. Vet. Parasitol. 3, 203-212.

Tafuri, Wg.L., Santos, R.L., Arantes, R.M.E., Gonçalves, R., Melo, M.N., Michalik, M.S.M., Tafuri, W.L., 2004. An alternative immunohistochemical method for detecting Leishmania amastigotes in paraffin-embedded canine tissues. J. Immunol. Methods 292, 17-23.

Veress, B., Omer, A., Satir, A.A., El Hassan, A.M., 1977. Morphology of the spleen and lymph nodes in fatal visceral leishmaniasis. Immunology 33, 605-610. 